\title{
Laparoscopic Removal of Left Non-Communicated Horn and Left Salpingectomy
}

\author{
Felemban A*, Alharbi U, Alblewi R, Alanazi H, Al \\ Anazi H, Al Beaiji $M$ and Alkheelb A \\ Department of Obstetrics \& Gynecology, King Abdullah \\ International Medical Research Center/King Saud bin \\ Abdul-Aziz University for Health Sciences, Saudi Arabia \\ *Corresponding author: Felemban A, Department \\ of Obstetrics \& Gynecology, Reproductive Medicine, \\ King Abdullah International Medical Research Center/ \\ King Saud bin Abdul-Aziz University for Health Sciences, \\ Hospital - NGHA, Riyadh, Saudi Arabia
}

Received: May 30, 2018; Accepted: J une 30, 2018; Published: August 06, 2018

\section{Introduction}

Uterine malformations are rare congenital abnormalities located within the female genital tract. The incidence of uterine malformation has been reported to be 0.1 and 3\% [1]. However, the incidence rate is not well defined, as many patients with this condition are asymptomatic. A classification system based on the degree of abnormal development was used to separate the anomalies into groups. Numerous characteristics were evaluated, including uterine absence and the presence of a unicornuate uterus, rudimentary uterine horn, blind uterine horn and symmetrical double uterus.

The unicornuate uterus caused by normal maturation of one müllerian duct with absent development of the contralateral duct. In cases where the contralateral Müllerian duct develops only partially, the unicornuate uterus is associated with a rudimentary horn, which may be cavitated or not cavitated and may or may not communicate with the unicornuate uterus.

The American Society of Reproductive Medicine (ASRM) classifies Unicornuate uteri into four groups:

1. A unicornuate uterus with a communicating rudimentary horn.

2. A unicornuate uterus with a non-communicating rudimentary horn.

3. A non-cavitated unicornuate uterus with a noncommunicating rudimentary horn.

4. An isolated unicornuate uterus [2].

The unicornuate uterus with non communicating rudimentary horn is the most common type. Complications of the rudimentary horns include endometriosis, primary infertility, hematometra, and associated renal anomalies [3]. This condition is often asymptomatic due to the lack of functional endometrium. However, when the horn is lined with functional endometrium, the resulting obstructed menstrual flow may cause severe cyclic pelvic pain shortly after menarche. The marked lower abdominal and pelvic pain may lead to the need for an ultrasound, computed tomographic scan, or magnetic resonance imaging, which demonstrates a pelvic mass. For these reasons, the diagnosis often made late. As a result, the diagnosis of complications is also late.

The traditional surgical approach to treatment of this problem has been through laparotomy and removal of the dilated non communicating horn. However, at least in the adult, a number of reported cases have now been treated successfully by laparoscopic removal [4]. The first laparoscopic removal of a rudimentary horn was performed in 1990 [4]. Since then, 17 single cases have been reported in the literature [5-15] as case reports, with no larger series. We present a case of primary laparoscopic removal in an adolescent, giving further evidence that it may be worthwhile to consider this approach in this age group also.

\section{Case Presentation}

19 year old lady, presented complaining of lower abdominal pain associated with severs dysmenorrhea and regular period. Examination within normal. Investigation normal laboratory. Pelvic ultrasound of bicorunate uterus with left hematometria $10.8 \times 5.5 \mathrm{~cm}$, right horn normal.

Patient then underwent MRI of abdomen and pelvis which showed uterine anomalies of bicorunate uterus, Left kidney not seen, right kidney normal.

Patient afterword underwent a diagnostic laparoscopy and hysteroscopy which showed there was small normal size right uterine horn + normal tube, ovary, rudimentary horn on left side + hydrosalpenix (14weeks size) + normal left ovary.

The patient was planned for operative laparoscopic left horn resection, received Lupron $3.7 \mathrm{mg}$ injection. Laparoscopic removal of left non-communicated horn with left salpingectomy. The left horn was enlarged as well as the left fallopian tube. The lower segment of the rudimentary horn looks like cervix. By using cautery salpingectomy was done. The left uterine rudimentary horn was attached to the right normal horn by broad muscular base which a small stump 
from it around $2 \mathrm{~cm}$ was left to strengthen the lateral uterine wall in case of future pregnancy the rudimentary horn was removed and sent for Histopathology which revealed pelvic adhesion biopsy consistent with endometriosis, left uterine horn showed endometrial lined partial thickness myometrium with separate endometrial like nodules. The cervical extensional part showed smooth muscle with fibrous septate, there was no cervical mucosa.

\section{Discussion}

Unicornate uterus with a rudimentary horn is the most uncommon uterine anomaly of the female genital tract [1]. The incidence of uterine anomalies in a fertile population is reported to be around 3.2\% [1]. It has an estimated frequency of one in 100,000 among the fertile female population [2]. This anomaly results from the abnormal maturation of one Müllerian duct with the normal development of the contralateral one [3]. This condition is often asymptomatic due to the lack of functional endometrium. However, when the horn is lined with functional endometrium, the resulting obstructed menstrual flow may cause severe cyclic pelvic pain shortly after menarche [4]. The marked lower abdominal and pelvic pain may lead to the need for an ultrasound, computed tomographic scan, or magnetic resonance imaging, which demonstrates a pelvic mass [5].

In cases where the contralateral Müllerian duct develops only partially, the unicornuate uterus is associated with a rudimentary horn, which may be cavitated or not cavitated and may or may not communicate with the unicornuate uterus [6]. A cavitated Non-communicating rudimentary horn (class II B according to the American Fertility Society [AFS] classification of Müllerian anomalies), can be found in about 20\%-25\% of women with unicornuate uterus [6]. There is no scientific evidence demonstrating that removing the rudimentary horn improves the reproductive outcome, although there are anecdotal reports regarding a positive effect on recurrent abortion [7]. However, a surgeon skilled in endoscopy surgery may become involved. In the adolescent, the traditional surgical approach to treatment of this problem has been through laparotomy and removal of the dilated Non-Communicating horn [8]. However, at least in the adult, a number of reported cases have now been treated successfully by laparoscopic removal. There are few cases published about unicorn ate uterus with rudimentary horn.

\section{References}

1. Simon C, Martinez L, Pardo F, Tortajada M. Pellicer A, Mullerian defects in women with normal reproductive outcome. Fertility and Sterility. 1991; 56: 1192-1193.

2. Atmaca R, Germen AT, Burak F, Kafkasli A. Acute abdomen in a case with noncommunicating rudimentary horn and unicornuate uterus. JSLS. 2005; 9: $235-237$.

3. Fedele L, Bianchi S, Zanconato G, Berlanda N, Bergamini V. Laparoscopic removal of the cavitated noncommunicating rudimentary uterine horn: surgical aspects in 10 cases. Fertil Steril. 2005; 83: 432-436.

4. Speroff L, Glass RH, Kase NG. Clinical GynecologicEndocrinology and Infertility. 6th ed. Baltimore, Md: Lippincott, Williams \& Wilkins; 1999: 148.

5. March CM. Hysteroscopy and the uterine factor in infertility. In: Lobo RA, Mishell DR, Paulson RJ, Shoupe D, eds. Mishells'Textbook of Infertility, Contraception, and Reproductive Endocrinology. 4th ed. Malden, Mass: Blackwell Science. 1997; 580-603.

6. American Fertility Society. The American Fertility Society classification of adnexal adhesions, distal tubal occlusion, tubal occlusion secondary to tubal ligation, tubal pregnancies, Mullerian anomalies andintrauterine adhesions. Fertil Steril. 1998; 49: 944 -955.

7. Fedele L, Bianchi S, Tozzi L, Marchini M, Busacca M. Fertility in women with unicornuate uterus. Br J Obstet Gynecol. 1995; 102: 1007-1009.

8. Soundararajan V, Rai J. Laparoscopic removal of a rudimentary uterine horn during pregnancy: Case report. J Reprod Med. 2000; 45: 599-602.
Austin J Clin Case Rep - Volume 5 Issue 2 - 2018 ISSN : 2381-912X | www.austinpublishing group.com Felemban et al. (C) All rights are reserved
Citation: Felemban A, Alharbi U, Alblewi R, Alanazi H, Al Anazi H, Al Beaiji M, et al. Laparoscopic Removal of Left Non-Communicated Horn and Left Salpingectomy. Austin J Clin Case Rep. 2018; 5(2): 1133. 